\title{
Inflammatory bowel disease: a beast of burden
}

\author{
Dawn Farrell
}

School of Nursing and Midwifery, UCC

\section{Introduction}

Imagine having to empty your bowel eight or ten times a day and experiencing constant panic and fear about the location of toilet facilities. Imagine experiencing constant tiredness that impacts on all aspects of your life including work, family and social life. These are just two examples of experiences commonly suffered by individuals with a condition called inflammatory bowel disease. These people are burdened with symptoms which impact on their daily lives. This research aims to provide healthcare professionals with an understanding of the extent to which individuals with inflammatory bowel disease experience symptom burden and to identify what symptoms are most problematic.

\section{Background}

Crohn's disease and ulcerative colitis collectively termed as inflammatory bowel disease are complex disorders. In the United Kingdom, collectively Crohn's disease and ulcerative colitis affects approximately one person in every 250 of the population. Ulcerative colitis affects up to 120,000 people in the UK, or every 1 in 500 people. On the other hand, Crohn's disease affects up to 60,000 people, or 1 in 1,000 people. The prevalence and incidence of inflammatory bowel disease in Ireland remains unknown, although it is estimated to affect approximately 25,000 people. The cause of inflammatory bowel disease is unclear. Science suggest that it is as a result of an immune reaction of the body against its own intestinal tissue. Presentation of the illness can occur at any age; however, typical onset occurs in the third to fourth decade of life. Men and women tend to suffer equally.

Inflammatory bowel disease is characterised by unpredictable periods of active and inactive disease states. The cardinal symptoms experienced by individuals with inflammatory bowel disease include diarrhoea, abdominal pain, weight loss and rectal bleed, particularly during periods of active disease. It is also now known that individuals with Crohn's disease and ulcerative colitis continue to experience a number of other symptoms during periods of apparent disease inactivity. For example, individuals report experiencing fatigue, abdominal cramping, loss of appetite and bloating. Research has shown that many 
individuals also experience psychological symptoms such as fear, anxiety and worry associated with their condition. The occurrence and duration of relapsing and remitting episodes and the frequency and severity of symptoms experienced over the course of the illnesses can vary from individual to individual. Moreover, individuals with inflammatory bowel disease are often treated with potent drug therapies, including steroids, which can additionally result in many undesired side-effects. These side-effects, coupled with the debilitating nature of the disease, can potentially impose significant symptom burden, thus limiting quality of life and functional status.

\section{What is symptom burden?}

Symptom burden is a distinct concept in symptom experience that takes into account the physiological and psychological functioning of the individual. It is a subjective, dynamic concept that is, it is the patient's own perception of a constantly changing experience. Symptom burden is quantifiable, in that it is a composite or combined measure of symptom severity, frequency and distress felt by individuals as a result of symptoms.

In summary, symptoms experienced by individuals with inflammatory bowel disease have the potential to impose significant symptom burden. However, a review of the literature highlights a paucity of research surrounding this concept in individuals with inflammatory bowel disease. Therefore, the aim of this research is to explore the symptom burden experiences of individuals with inflammatory bowel disease. Two open-ended questions on a measure administrated as part of the first phase of a larger two-phased study were used.

\section{Symptom burden experiences of individuals with inflammatory bowel disease}

Individuals with inflammatory bowel disease experience a substantial number of symptoms over the course of their illness which may contribute to symptom burden. Preliminary results from this research identified specific symptoms of greatest burden and the consequential reasons for this from a sample of 185 participants.

\section{Symptoms of greatest burden}

Each participant was asked to identify the three most burdensome symptoms experienced on a daily basis. A range of one to three symptoms were reported by each of the 185 participants. A total of 50 different symptoms were identified by participants as the most burdensome symptoms experienced, including both physical and psychological symptoms. Of 
the participants who reported their three most burdensome symptoms (185 respondents), the most commonly reported were lack of energy or fatigue (73 respondents), pain (72 respondents), of which 13 participants specifically reported joint pain (13 respondents) and diarrhoea (65 respondents). A number of individuals communicated bowel urgency (43 respondents) and the constant need to go to the toilet or bowel frequency (26 respondents) as most burdensome. Abdominal/stomach cramps (34 respondents), bloating ( $\mathrm{n}=$ 18 respondents), passing wind/flatulence (15 respondents), lack of appetite (10 respondents), sleep disturbances ( 9 respondents) and rectal bleeding (9 respondents) were also demonstrated as contributing to significant symptom burden. Although less prevalent, psychological symptoms such as worrying, fear, feeling irritable, nervousness, depression, anxiety, and 'emotional problems' were detailed among the most burdensome experienced.

\section{Reasons why symptoms are burdensome}

The reasons why symptoms caused the greatest burden was explored and a number of explanations were provided by participants.

\section{Limits social activity}

Diarrhoea, bowel urgency and bowel frequency were commonly described as limiting social activity and thus augmenting the burden associated with the symptom. Individuals presented their social life as "non existent", "terrible", and "restrictive". Their reluctance to socialize was often attributed to the constant need to be near a toilet due to bowel frequency and urgency. Participants have to continually plan their day ahead so that they would be aware of the nearest toilet facility at all times. As a result, feelings of nervousness, worry, fear and panic were associated with leaving home and being in strange places. Additionally, participation in physical activity and sports was restricted or impossible due to the debilitating effect of fatigue.

\section{Negatively impacts on working life}

Pain and lack of energy was described as affecting the working life of individuals, particularly self-employed individuals or those who worked long hours or had physical jobs. In particular, lack of energy caused difficulties at work such as motivational issues and often resulted in absenteeism from work. Tiredness was depicted as a constant struggle with regard to work. 


\section{Negatively impacts on family life}

Impact on family life was also evident from the participants' responses, in particular from the burden related to lack of energy. Parents who experience burdensome symptoms expressed difficulties in caring for their children and an inability to enjoy family life. One mother reported lack of energy leaving her

"feeling as if I'm only a burden on my family and they'd be better off without me"

\section{Diet restriction}

Diet was another struggle for individuals with inflammatory bowel disease as often pain and diarrhoea is experienced following eating, thus contributing to the burden associated with these symptoms. Lack of appetite and mouth ulcers often meant individuals were unable to enjoy their food. Restrictions on diet were imposed as a result.

\section{Attributed to other symptoms}

Individuals felt that symptoms were often interrelated and experiencing a number of debilitating symptoms concurrently resulted in greater symptom burden. One participant stated:

"Abdominal pain, diarrhoea and emotional problems are interconnected. Pain and diarrhoea go hand in hand leading to you feeling down"

This comment depicted the effects of physical symptoms on the psychological state of individuals. For example, individuals experiencing passing blood in their stool reported feeling frightened. Bowel urgency and incontinence were associated with worry and anxiety. Pain, due to its difficulty to manage, was reported to substantially impact on one's mood. Lack of sleep was reported to lead to feeling irritable and cranky. Bloating impacted on self-esteem and body image. Embarrassment was one of the most commonly reported psychological reason why symptoms contribute to burden. Stomach gurgling, flatulence, bowel urgency, diarrhoea, foul smelling bowel motion, rash and sweating were all described as embarrassing, thus contributing to greater burden.

Likewise, psychological symptoms appeared to be linked with physical symptoms. Stress and worry were reported to cause diarrhoea and lack of appetite. One participant stated that:

"anxiety causes the flare ups of Crohn's"

Overall, reports by individuals participating in this research expressed that symptoms wear away at them and slowed them down in their day to day life, thus amplifying the symptom burden experience. Individuals expressed that they lacked control over their lives and felt 
that symptoms "had a hold on their life" resulting in them not being able to live their lives as they wish.

\section{Conclusion}

In conclusion, the findings thus far have illustrated a substantial number of symptoms that individuals consider burdensome. The complex impact of these burdensome symptoms offers insight into the reasons contributing to symptom burden. This knowledge will help healthcare professionals to be more sensitive to individual's needs and assist supporting them in managing their symptoms. A goal of care for all healthcare professionals is to prevent suffering and improve quality of life, thus highlighting the importance of this research.

Moreover, these findings will support the findings from the first phase of my $\mathrm{PhD}$ study, which quantitatively measures the level of symptom burden experienced by individuals with inflammatory bowel disease. Additionally, these findings will inform the second phase of the study which will qualitatively explore the self-management strategies used to control such burdensome symptoms. It is anticipated that this research will provide data for the future development of nurse-led interventions aimed at reducing the symptom burden experienced and improve self-management strategies.

Dawn Farrell is a 2nd PhD student in Catherine McAuley School of Nursing and Midwifery, Brookfield Health Sciences Complex, under the supervision of Professor Eileen Savage and Professor Geraldine McCarthy, Catherine McAuley School of Nursing and Midwifery, University College Cork, Cork, Ireland. 